\title{
Combined Local and Centralized Voltage Control in Active Distribution Networks
}

\author{
Hamid Soleimani Bidgoli, Student Member, IEEE, and Thierry Van Cutsem, Fellow Member, IEEE
}

\begin{abstract}
A two-level real-time voltage control scheme is proposed to keep voltages within specified limits in distribution grids using Distributed Generation Units (DGUs). It combines a local and a centralized control of their reactive powers. The local control provides fast response after a disturbance, reducing its impact and enhancing voltage quality. The centralized control uses measurements collected throughout the network to bring the voltages inside tighter limits and balance the various DGU contributions. To this purpose, it adjusts in a coordinated way their reactive power set-points, taking into account the local controls. This discrete control solves at each time step a multitime-step constrained optimization inspired of Model Predictive Control. The method effectiveness is demonstrated on a 75-bus test system hosting 22 DGUs.
\end{abstract}

Index Terms-Active distribution network, real-time control, voltage control, model predictive control.

\section{INTRODUCTION}

$\mathbf{T}$ HE continuous growth of renewable energy injected into medium-voltage distribution systems is expected to create new operational problems. Over- or under-voltages are some of the main issues caused by intermittent production of the dispersed units. It can be partly mitigated in operational planning stage [1]. However, in real-time, the system can be driven to insecure situations, for instance due to unforeseen incidents. The abnormal voltages can be tolerated for a short period of time, but it is desirable to bring them within limits as soon as possible. In the context of active distribution networks, the DGUs can be used as controls to meet this objective, which is an attractive alternative to (or at least a way to postpone) network reinforcement.

Over the last decade, a growing attention has been paid to real-time control of reactive powers and voltages in distribution grids hosting a significant number of DGUs, as testified for instance by the recent survey in [2].

The methods can be broadly categorized according to the control architecture: local (or decentralized) [3]-[5]; agentbased [6]-[9]; centralized [7], [10]-[15]; and multi-layer [16][18]. Alternatively, the methods can be classified as modelfree [3], [4], model-based [6], [7], [7]-[18], or methods with limited knowledge of the system [5].

The most widely used, and also simplest approach is local control. It is already present in a number of grid codes [3]. For example, in [4] a decentralized scheme was proposed to

Hamid Soleimani Bidgoli is with the Dept. of Electrical Engineering and Computer Science, University of Liège, Belgium; e-mail: h.soleimani@ulg.ac.be.

Thierry Van Cutsem is with the Fund for Scientific Research (FNRS) at the Dept. of Electrical Engineering and Computer Science, University of Liège, Belgium; e-mail: t.vancutsem@ulg.ac.be. control DGU terminal voltages. The units operate in power factor mode, when no violation is observed, and in voltage control mode, when their terminal voltages exceed upper/lower limits. Furthermore, if the voltage problem cannot be solved by the sole reactive power adjustment, the DGUs active powers are also curtailed, in the last resort. To obtain more precise adjustment of DGUs in voltage control mode, the work was further extended in [5] by including the sensitivities of voltages to DGU outputs.

As an alternative with more information exchange, an agentbased scheme was proposed in [6] in which, by using locally collected measurements, the distributed controllers mitigate the voltage violations and, when needed, initiate an additional reactive power support request from the neighbouring controllers. Using an Optimal Power Flow (OPF), Ref. [7] discussed the impact of centralized and distributed voltage control schemes on potential penetration of dispersed generation. A distributed architecture, comprising several cooperative smart agents, was proposed in [8] to solve the voltage regulation problem. After obtaining the operating values, each agent optimizes its own design. In Ref. [9] an agent-based system was proposed to control the DGUs in a low-voltage grid in a distributed manner. That work also considered different local reactive power characteristics and compared the corresponding system behaviours.

Centralized, coordinated control of DGUs relies on a proper communication infrastructure to collect measurements and send control corrections at regular time intervals. In this context, Ref. [10] proposed a control scheme relying on the concept of Model Predictive Control (MPC). This involves solving repeatedly a multi-time-step constrained optimization problem with a sensitivity model to predict the system evolution. The controller coordinates the power outputs of DGUs and the voltage set-point of the transformer Load Tap Changer (LTC) to smoothly bring the unacceptable voltages inside specified bounds. The approach was extended in [11], [12] to handle congestions and track desired production schedules. Compared to standard open-loop OPF, the closed-loop nature of MPC offers significant advantages in terms of robustness to model inaccuracies, component failures, etc. [13], [14]. Considering the uncertain nature of wind, the work in [15] extended a deterministic OPF to a risk-based OPF approach subject to probabilistic constraints. The centralized controller aimed at maximizing the exploited wind energy and reducing control actions.

More recently, using a dynamic model of the system, Ref. [16] suggested a multi-layer control structure. At the upper level, a static OPF computes reference values of reactive 


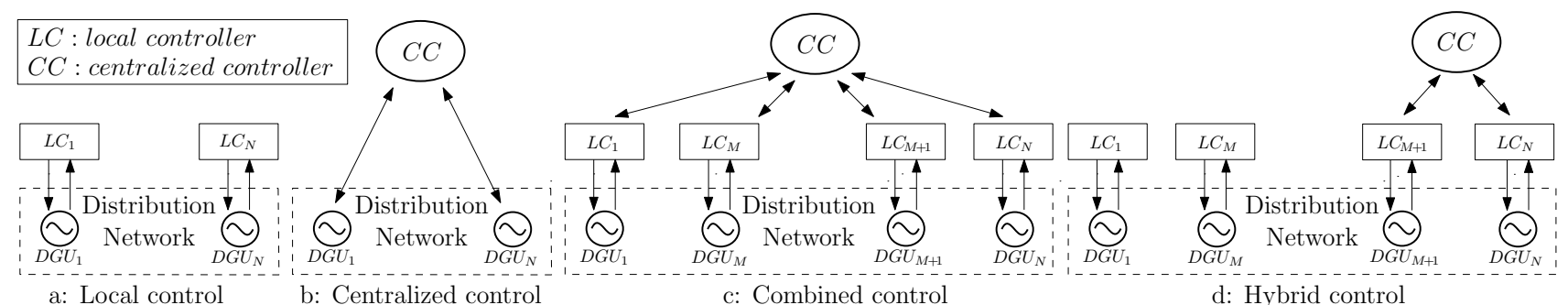

Fig. 1. Voltage control architectures

powers. The latter are communicated to the next layer, an MPC-based centralized controller, which handles the operation constraints. A combined scheme was proposed in [17] in which local controllers provide fast responses and a centralized controller uses power injection predictions for the next hours to update the droop parameters of the local controllers, ensuring that the voltages are kept within their limits in the forecast time interval. Reference [18] proposes a two-stage strategy for distributed energy storage management. In day-ahead, the optimized battery charge/discharge schedules are calculated centrally. Then, they are communicated to local controllers for further short-term adjustments, when approaching real-time.

This paper focuses on real-time correction of abnormal voltages. The proposed control has two levels, local and centralized, with the objective of combining the respective advantages of both schemes:

- in a couple of seconds, the local level provides the fast response to a disturbance, reducing its impact and enhancing voltage quality;

- in some tens of seconds, the central level coordinates the various DGUs in order to refine the local corrections and balance the various DGU contributions;

- the local level acts as back-up in case of communication failures between the DGUs and the centralized controller, which adds to the overall reliability.

The paper is organized as follows. Section II outlines various voltage control architectures. Section III and IV detail respectively the lower and the upper level of the proposed scheme. Simulation results are reported in Section V, while Conclusions are offered in Section VI.

\section{VOLTAGE CONTROL ARCHITECTURES}

Different control schemes can be considered in a distribution system taking into account the practical needs, technical limitations of the controllable devices (mainly DGUs, possibly shunt capacitors) and regulatory policies. Two broad categories are the local and the centralized architectures, depicted in Figs. 1.a and 1.b, respectively. Local control is implemented inside the equipment and adjusts the reactive power of each DGU based on local measurements only, essentially the terminal voltage. The measurements are collected without communication delay. Thus, a fast reaction is obtained while no communication infrastructure is required. A centralized controller, on the other hand, periodically gathers measurements and sends set-point corrections to the controlled devices. Having

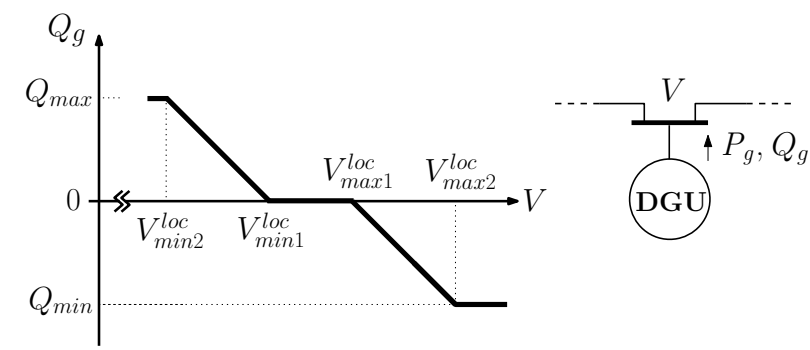

Fig. 2. Local level: steady-state $V Q$ characteristic

a wider view of the system, this controller is able to share the corrective efforts over multiple DGUs.

A potentially attractive scheme consists of combining local and centralized controllers, for greater flexibility. This approach, sketched in Fig. 1.c, will be the main focus of this paper. At the local level, the DGUs are equipped with continuous controllers adjusting reactive powers in response to terminal voltage variations. At the upper level, a discrete-time controller receives voltage and reactive power measurements and adjusts set-points of the local controllers in order to improve the overall system behaviour.

In some distribution systems, the deployment of the upper level of the proposed scheme might not be feasible or affordable, for instance with existing and/or conventional DGUs. Hence, the hybrid control architecture shown in Fig. 1.d can be envisaged, in which the combined control scheme steers only a subset of DGUs, the rest of them are under local control only. In this case, it is important for the centralized controller to anticipate the reactions of all local controllers.

\section{LOWER-LEVEL: LOCAL CONTROL}

\section{A. Reactive power control of a $D G U$}

In steady state the reactive power output of a DGU under local control varies according to the piecewise linear $V Q$ characteristic shown in Fig. 2. Such characteristic was proposed for instance in [19]-[22]. As long as the measured terminal voltage lies within the dead-band $\left[V_{\min 1}^{l o c}, V_{\max 1}^{l o c}\right]$, the produced reactive power $Q_{g}$ is kept at zero, which is usually preferred to minimize DGU internal losses. Outside the above mentioned dead-band, the DGU reacts to over or under-voltage by consuming or producing reactive power, respectively. The DGU is locked at its maximum reactive power production $Q_{\max }$ (resp. consumption $Q_{\min }$ ), if the voltage stays below $V_{\min 2}^{l o c}\left(\right.$ resp. above $\left.V_{\max 2}^{l o c}\right)$. 


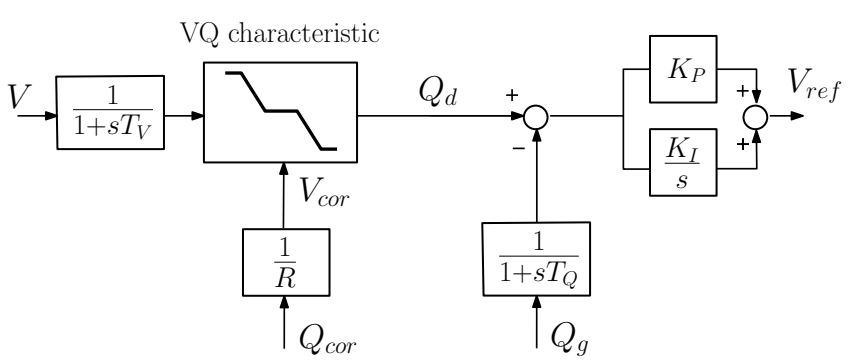

Fig. 3. Local level: generic model of DGU reactive power control

A generic model of DGU reactive power control is shown in Fig. 3. The terminal voltage $V$ and the generated reactive power $Q_{g}$ are measured, with the corresponding time constants $T_{V}$ and $T_{Q}$. The desired reactive power output $Q_{d}$ is given by the $V Q$ characteristic using the measured voltage. The difference between $Q_{d}$ and the measured reactive power is processed by a Proportional-Integral controller. The output $V_{r e f}$ is the terminal voltage reference in a power-electronics based DGU, or the field voltage in a synchronous generator. Let us stress that the above model does not encompass all practical voltage control schemes (see e.g. [23]), but it serves the main purpose of this paper.

For coordination purposes the local controller receives a reactive power correction $Q_{c o r}$ updated and sent at discrete times by the centralized controller. This correction results in a shift of the $V Q$ characteristic, as detailed in the next section.

The change of reactive power output of one DGU affects the voltages at other buses, including DGU buses. Assuming that these DGUs are not operating in the dead-band of their $V Q$ characteristics, they will react to the voltage change by also adjusting their reactive powers. The interactions between locally controlled DGUs are such that the reactive power increase of a DGU causes reactive power decreases of other DGUs. The response time is at most a couple of seconds.

\section{B. Correction from the centralized controller}

As suggested in Fig. 3, the correction $Q_{c o r}$ received from the centralized controller modifies the $V Q$ characteristic. Namely, the piecewise linear characteristic is shifted parallel to the $V$ axis. The purpose of this is better explained with an example.

Figure 4 shows an over-voltage situation and the subsequent actions at both levels to remove the violation. The initial operating point of the DGU, shown with a black dot, is at the intersection of the DGU and network $V Q$ characteristics. In the example of Fig. 4.a, the voltage lies in the dead-band; therefore, initially, the DGU operates at unity power factor. Under the effect of a disturbance, the network characteristic changes and the DGU terminal voltage exceeds the upper limit $V_{\max 1}^{l o c}$. The circle in Fig. 4.b shows the situation with no control. Although the violation is partly corrected by a first and fast reaction of the local controller (black dot in Fig. 4.b), the voltage is still above the upper voltage limit $V_{\max }^{c n t}$ monitored by the centralized controller. The latter computes a sequence of corrections $\Delta Q_{c o r}$ and sends them to the local controller.
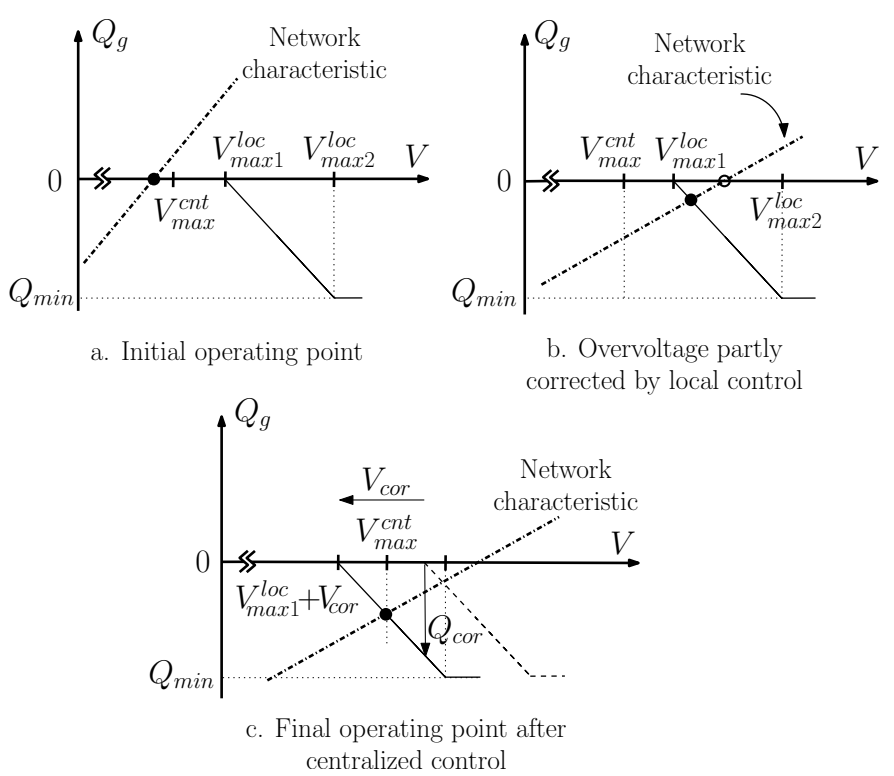

Fig. 4. Example of over-voltage correction by local and centralized controls (only high voltage part of the characteristic is shown). The smaller slope of the VQ curve in sub-figures 4.b and 4.c compared to 4.a corresponds to a larger Thévenin impedance seen by the DGU

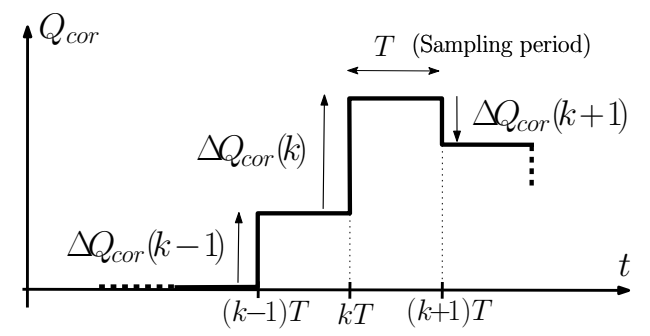

Fig. 5. Local level: cumulation of reactive power corrections received from the centralized controller

At the lower level, the successively received corrections are cumulated as shown in Fig. 5, where $k$ is the discrete time, $T$ the sampling period and $Q_{c o r}$ the cumulated (or discrete integral) correction. The latter is used to shift the $V Q$ characteristic as shown in Fig. 4.c. Assuming operation on the sloping part of the $V Q$ characteristic, the voltage shift $V_{\text {cor }}$ corresponding to a given value of $Q_{c o r}$ is:

$$
V_{\text {cor }}=\frac{Q_{c o r}}{R}
$$

where $R$ is the local droop of the $V Q$ characteristic.

The upper level keeps on sending $\Delta Q_{c o r}$ corrections until the voltage is brought at the $V_{\max }^{c n t}$ limit, as illustrated by the black dot in Fig. 4.c.

Note that the cumulated correction $Q_{c o r}$ received from the centralized controller is different from the DGU effective reactive power change, as seen from Fig. 4.c. A linear relation between both can be used, as detailed in Section IV-B.

Note also that different voltage limits are specified in the local and centralized controls. Local control aims at mitigating the voltage excursion in the very first seconds after a disturbance. The centralized control acts only if the resulting voltage exceeds the limit $V_{\max }^{c n t}$. This is the case if $V_{\max }^{c n t}<V_{\max 1}^{l o c}$. 
A similar behaviour is obtained in case of under-voltage.

With the above correction, the expression of the " $V Q$ characteristic" block in Fig. 3 can be detailed as follows:

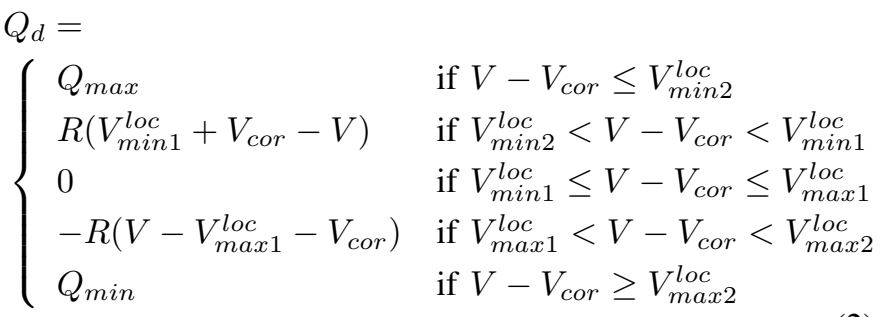

where the various voltage thresholds are defined in Fig. 2, and the same droop $R$ has been considered for under- and over-voltage, for simplicity.

\section{UPPER LEVEL: CENTRALIZED CONTROL}

The aim of the upper-level controller is to bring voltages inside tighter limits and balance the various DGU contributions. It relies on measurements collected throughout the network.

For clarity, the formulation in Sections IV-A and IV-B involves only the corrections $\Delta \boldsymbol{Q}_{\text {cor }}$ sent to lower level, while extensions to DGU active powers and LTC voltage set-point are discussed in Sections IV-C and IV-D, respectively.

In all derivations, the three-phase distribution network is assumed to operate in balanced conditions.

\section{A. MPC formulation}

As already mentioned, a multi-time-step optimization is at the heart of the MPC used at the centralized level.

At a discrete time $k$, the objective is to minimize over the next $N_{c}$ steps the deviations of the DGU reactive powers, $\boldsymbol{Q}_{g}(k+i)$, from their last measured values, $\boldsymbol{Q}_{g}^{m}(k)$ :

$$
\min _{\boldsymbol{Q}_{g}, \boldsymbol{V}, \boldsymbol{\varepsilon}, \Delta \boldsymbol{Q}_{g}, \Delta \boldsymbol{Q}_{\text {cor }}} \sum_{i=0}^{N_{c}-1}\left\|\Delta \boldsymbol{Q}_{g}(k+i)\right\|_{\boldsymbol{W}_{Q}}^{2}+\|\varepsilon\|_{\boldsymbol{S}}^{2}
$$

where $\left(i=0, \ldots, N_{c}-1\right)$ :

$$
\Delta \boldsymbol{Q}_{g}(k+i)=\boldsymbol{Q}_{g}(k+i)-\boldsymbol{Q}_{g}^{m}(k)
$$

and $\boldsymbol{W}_{Q}$ is a diagonal matrix allowing to give different weights to different DGUs. The second term in (3) involves the slack variables $\varepsilon$ aimed at relaxing the inequality constraints (detailed hereafter) in case of infeasibility. Matrix $\boldsymbol{S}$ is also diagonal with large diagonal elements to force the constraints. The quadratic $\left(L_{2}\right)$ norm in (3) tends to spread the whole control effort over a larger number of DGUs among those that can help correcting the voltage problem.

The minimization is subject to the linearized relation between $\Delta \boldsymbol{Q}_{g}$ and the control variables $\left(i=0, \ldots, N_{c}-1\right)$ :

$$
\Delta \boldsymbol{Q}_{g}(k+i)=\boldsymbol{S}_{Q Q} \Delta \boldsymbol{Q}_{\text {cor }}(k+i)
$$

as well as the linearized predicted evolution of voltages over the future $N_{p}$ steps $\left(i=1, \ldots, N_{p}\right)$ :

$$
\begin{gathered}
\boldsymbol{V}(k+i)=\boldsymbol{V}^{m}(k)+\boldsymbol{S}_{V Q} \Delta \boldsymbol{Q}_{g}(k+i-1) \\
\boldsymbol{I}(k+i)=\boldsymbol{I}^{m}(k)+\boldsymbol{S}_{I Q} \Delta \boldsymbol{Q}_{g}(k+i-1)
\end{gathered}
$$

where $\boldsymbol{V}(k+i)$ and $\boldsymbol{I}(k+i)$ are the vector of predicted bus voltages and branch currents at time $k+i$. The prediction is initialized with the last gathered measurements $\boldsymbol{V}^{m}(k)$ and $\boldsymbol{I}^{m}(k) . \boldsymbol{S}_{Q Q}$ and $\boldsymbol{S}_{V Q}$ are sensitivity matrices whose derivation is detailed in the next section, and $\boldsymbol{S}_{I Q}$ relates the branch current variation to DGU reactive power changes [12]. The use of the static transition model (6) and (7) is justified by the fast response of the power electronics based DGUs compared to the MPC sampling time.

Finally, the following inequality constraints are imposed:

$$
\varepsilon=\left[\varepsilon_{1} \varepsilon_{2}\right] \geq \mathbf{0}
$$

$$
\begin{aligned}
& \text { for } i=1, \ldots, N_{p}: \\
& \qquad \begin{array}{l}
\left(-\varepsilon_{1}+V_{\min }^{c n t}\right) \mathbf{1} \leq \boldsymbol{V}(k+i) \leq\left(V_{\max }^{c n t}+\varepsilon_{2}\right) \mathbf{1} \\
\boldsymbol{I}(k+i) \leq \boldsymbol{I}_{\max }
\end{array}
\end{aligned}
$$

for $i=0, \ldots, N_{c}-1$ :

$$
\begin{gathered}
\boldsymbol{Q}_{g}^{\min }(k) \leq \boldsymbol{Q}_{g}(k+i) \leq \boldsymbol{Q}_{g}^{\max }(k) \\
\Delta \boldsymbol{Q}_{g}^{\min } \leq \boldsymbol{Q}_{g}(k+i)-\boldsymbol{Q}_{g}(k+i-1) \leq \Delta \boldsymbol{Q}_{g}^{\max }
\end{gathered}
$$

where 1 denotes a unit vector, $\boldsymbol{Q}_{g}^{\min }, \boldsymbol{Q}_{g}^{\max }, \Delta \boldsymbol{Q}_{g}^{\min }$ and $\Delta Q_{g}^{\max }$ are the lower and upper limits on DGU reactive powers and on their rates of change. In Eq. (12), $\boldsymbol{Q}_{g}(k-1)$ is set to $\boldsymbol{Q}_{g}^{m}(k)$.

After the voltage, active and reactive power measurements have been received from the DGUs, and before the optimisation is solved, the bounds $\boldsymbol{Q}_{g}^{\min }$ and $\boldsymbol{Q}_{g}^{\max }$ in (11) are updated in accordance with the DGU capability curves [24], assuming that the active power will not change significantly over the control horizon. Thus the bounds vary with the discrete time $k$, but, for legibility, the dependency on active power and voltage is omitted in the notation.

1) Remark 1: The above formulation is similar to the one used in [11]. However, a significant difference lies in the fact that the objective (3) does not involve the deviations of control variables but $\Delta \boldsymbol{Q}_{g}$, which is a linear function of the control variables $\Delta \boldsymbol{Q}_{\text {cor }}$. This modification was required to properly account for the lower level control.

2) Remark 2: The formulation is such that, if all voltages and currents lie inside the limits defined by $(9,10)$, the obvious solution is $\Delta \boldsymbol{Q}_{\text {cor }}=\mathbf{0}$, i.e. no control is sent to the DGUs. Targeting this feasible set only, instead of specific voltage setpoints, yields good stability. Indeed it bears similarity with the terminal constraint-set method discussed in [25].

3) Remark 3: Different $\boldsymbol{W}_{Q}$ entries can be assigned to different DGUs, in order to favour some of them. The technology used in the plant, the type of contract with DGU owners, enabling the DSO to adjust the DGU outputs, etc. can be taken into account.

\section{B. Derivation of sensitivity matrices}

The sensitivity matrix $\boldsymbol{S}_{V Q}$ (resp. $\boldsymbol{S}_{Q Q}$ ) expresses how much the bus voltages (resp. the DGU reactive powers) change after a small change $\Delta \boldsymbol{Q}_{\text {cor }}$ of the control variables.

A graphic view is given in Fig.6, showing the voltage and reactive power changes induced a variation of $\Delta Q_{c o r}$ in one 
DGU. Assuming operation on the inclined part of the $V Q$ characteristic, the figure shows the resulting change of voltage $\Delta V$ and reactive power $\Delta Q_{g}$.

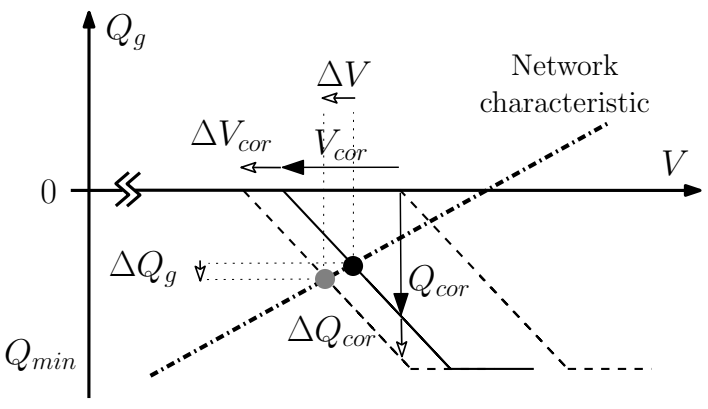

Fig. 6. Graphic representation of the effects of a small control change $\Delta Q_{c o r}$

The equation of the solid black line is:

$$
Q_{g}-Q_{c o r}=-R\left(V-V_{\max 1}^{l o c}\right)
$$

involving the already defined droop $R$. Considering small deviations denoted with $\Delta$, Eq. (13) gives:

$$
\Delta Q_{g}-\Delta Q_{c o r}=-R \Delta V
$$

This equation can be written in matrix form for all DGUs as:

$$
\Delta \boldsymbol{Q}_{g}=\Delta \boldsymbol{Q}_{\text {cor }}-\boldsymbol{R} \Delta \boldsymbol{V}
$$

where $\boldsymbol{R}$ is a diagonal matrix whose diagonal elements are the various droop coefficients.

The variations of bus voltages with the DGU reactive powers is given by:

$$
\Delta \boldsymbol{V}=\boldsymbol{S}_{V Q} \Delta \boldsymbol{Q}_{g}
$$

where $S_{V Q}$ can be obtained from the transposed inverse of the power flow Jacobian matrix. Alternatively, each column of the matrix can be computed by running a power flow calculation with one DGU reactive power slightly modified, and dividing the bus voltage variations by the reactive power variation considered (see e.g. [12] for more details).

By substituting (16) in (15), one easily obtains:

$$
\Delta \boldsymbol{Q}_{g}=\left(\boldsymbol{U}+\boldsymbol{R} \boldsymbol{S}_{V Q}\right)^{-1} \Delta \boldsymbol{Q}_{c o r}
$$

where $U$ is a unit matrix. The sought matrix is thus given by:

$$
\boldsymbol{S}_{Q Q}=\left(\boldsymbol{U}+\boldsymbol{R} \boldsymbol{S}_{V Q}\right)^{-1}
$$

The above calculation is made under the assumption that all DGUs operate on the sloping portion of their $V Q$ characteristics. This could be justified by the fact that, after a significant voltage disturbance, the DGUs contributing the most to local voltage control will have their operating points moved to those sloping portions. The fact remains, however, that for DGUs operating in their dead-band, Eq. (18) is inexact.

One option would be for the centralized controller to know the status of operation of each DGU and update $S_{Q Q}$ to reflect the changes. This would entail too much complexity. It is more appealing to work with a non-updated $\boldsymbol{S}_{Q Q}$ matrix and leave it to MPC to compensate for the error, taking advantage of its ability to operate with a somewhat inaccurate model.
More precisely, when the centralized controller assigns a correction $\Delta Q_{\text {cor }}$ to a DGU assuming implicitly that it operates on the sloping portion of its $V Q$ characteristic, while it lies (and remains) in its dead-band, the DGU does not respond with the expected additional reactive power. This will cause the MPC to repeat its requests at subsequent times. In the best case, the $V Q$ curve is enough shifted so that the DGU eventually operates on the sloping portion (and hence adjusts its reactive power). If those repeated attempts remain unsuccessful, other DGUs will be solicited and the voltage correction is likely to take some more time.

An example of this situation is presented in Section V.D.

\section{Extension to DGU active power control}

The DGU reactive powers are the preferred control means to correct abnormal voltages. In severe conditions where there are not enough DGUs, or they are not properly located in the network, the optimization problem (3)-(12) may become infeasible. This is easily detected by a nonzero value of $\varepsilon$, which gives a warning that other control means are needed, namely the voltage set-point of the LTC and, in the last resort, the DGU active powers. LTC control is discussed in the next section, while an extension that encompasses both active and reactive power controls is given hereafter.

Note that this extension is also needed when the initial problem is a thermal overload that cannot be solved by changing reactive powers only.

The objective function (3) becomes:

$\min \sum_{i=0}^{N_{c}-1}\left\|\Delta \boldsymbol{Q}_{g}(k+i)\right\|_{\boldsymbol{W}_{Q}}^{2}+\left\|\Delta \boldsymbol{P}_{g}(k+i)\right\|_{\boldsymbol{W}_{P}}^{2}+\|\varepsilon\|_{\boldsymbol{S}}^{2}$

where $\Delta \boldsymbol{P}_{g}$ is the vector of active power corrections:

$$
\Delta \boldsymbol{P}_{g}(k+i)=\boldsymbol{P}_{g}(k+i)-\boldsymbol{P}_{g}^{m}(k)
$$

and the entries of $\boldsymbol{W}_{P}$ are set much higher than those of $\boldsymbol{W}_{Q}$ in order to give priority to reactive power changes. Similar to $(11,12)$, the following constraints are imposed on active powers and their rates of change $\left(i=0, \ldots, N_{c}-1\right)$ :

$$
\begin{gathered}
\boldsymbol{P}_{g}^{\min }(k) \leq \boldsymbol{P}_{g}(k+i) \leq \boldsymbol{P}_{g}^{\max }(k) \\
\Delta \boldsymbol{P}_{g}^{\text {min }} \leq \boldsymbol{P}_{g}(k+i)-\boldsymbol{P}_{g}(k+i-1) \leq \Delta \boldsymbol{P}_{g}^{\max }
\end{gathered}
$$

The choice of $\boldsymbol{P}_{g}^{\max }(k)$ is discussed in [12].

The equality constraints (5)-(7) are extended as follows to account for active power changes:

$$
\begin{aligned}
& \Delta \boldsymbol{Q}_{g}(k+i)=\boldsymbol{S}_{Q Q} \Delta \boldsymbol{Q}_{\text {cor }}(k+i)+\boldsymbol{S}_{Q P} \Delta \boldsymbol{P}_{g}(k+i) \\
& \boldsymbol{V}(k+i)=\boldsymbol{V}^{m}(k)+\boldsymbol{S}_{V Q} \Delta \boldsymbol{Q}_{g}(k+i-1) \\
&+\boldsymbol{S}_{V P} \Delta \boldsymbol{P}_{g}(k+i-1) \\
& \boldsymbol{I}(k+i)=\boldsymbol{I}^{m}(k)+ \boldsymbol{S}_{I Q} \Delta \boldsymbol{Q}_{g}(k+i-1) \\
&+ \boldsymbol{S}_{I P} \Delta \boldsymbol{P}_{g}(k+i-1)
\end{aligned}
$$

The extended formulation including active power changes consists in minimizing the objective (19) subject to the constraints (8)-(12) together with (20)-(25).

The sensitivity matrix $\boldsymbol{S}_{V P}$ (resp. $\boldsymbol{S}_{I P}$ ) is determined similarly to $\boldsymbol{S}_{V Q}$ (resp. $\left.\boldsymbol{S}_{I Q}\right)$. The $\boldsymbol{S}_{Q P}$ matrix is derived as 
follows. The variations of bus voltages with the DGU active and reactive powers is now given by:

$$
\Delta \boldsymbol{V}=\boldsymbol{S}_{V Q} \Delta \boldsymbol{Q}_{g}+\boldsymbol{S}_{V P} \Delta \boldsymbol{P}_{g}
$$

Substituting this expression for $\Delta \boldsymbol{V}$ in (15) yields:

$$
\Delta \boldsymbol{Q}_{g}=\Delta \boldsymbol{Q}_{\text {cor }}-\boldsymbol{R} \boldsymbol{S}_{V Q} \Delta \boldsymbol{Q}_{g}-\boldsymbol{R} \boldsymbol{S}_{V P} \Delta \boldsymbol{P}_{g}
$$

which can be rewritten as:

$$
\left(\boldsymbol{U}+\boldsymbol{R} \boldsymbol{S}_{V Q}\right) \Delta \boldsymbol{Q}_{g}=\Delta \boldsymbol{Q}_{\text {cor }}-\boldsymbol{R} \boldsymbol{S}_{V P} \Delta \boldsymbol{P}_{g}
$$

from which the sought sensitivity matrices are obtained as:

$$
\begin{aligned}
\boldsymbol{S}_{Q Q} & =\left(\boldsymbol{U}+\boldsymbol{R} \boldsymbol{S}_{V Q}\right)^{-1} \\
\boldsymbol{S}_{Q P} & =-\left(\boldsymbol{U}+\boldsymbol{R} \boldsymbol{S}_{V Q}\right)^{-1} \boldsymbol{R} \boldsymbol{S}_{V P}
\end{aligned}
$$

\section{Extension to transformer ratio control}

Another option for voltage control consists of adjusting the ratio of the transformer connecting the distribution grid to transmission. If the transformer is equipped with an automatic LTC, its voltage set-point should be controlled instead of the tap position. As discussed in [10], either that set-point is treated as a control variable, or the LTC is assumed to operate independently, and its effects are anticipated through a correction term in the prediction (6).

However, increasing the number of tap changes reduces the LTC lifetime, and considering the higher accuracy and speed offered by power electronics-based DGUs, the latter are progressively preferred to actions on LTCs.

Hence, in this work, voltage correction is performed without the contribution of the LTC. If the latter had to be considered, it would be added to the controls with an associated "cost" significantly higher than that of DGU reactive powers, so that the latter are favoured by the optimization.

\section{E. Interactions between local and centralized controls}

As already explained a disturbance triggers a fast reaction of local controllers, followed by a slower corrections by the centralized controller. There are basically two cases:

- the measurements used by the centralized controller are collected after the DGU powers have reached (almost) steady state. In this case there is clear separation between local and centralized controls. Furthermore, the centralized controller will benefit from measurements that already reflect the contribution of local controls;

- the measurements used by the centralized controller are collected while the DGU powers are still evolving in response to the disturbance. The measurements are thus affected by these transients, which can be seen as noise, compensated by the closed-loop feature of the centralized controller.

\section{Simulation RESUltS}

\section{A. Test system and control settings}

The performance of the combined control is illustrated on a 75-bus, $11-\mathrm{kV}$ distribution network whose one-line diagram is shown in Fig.7 [26]. It is connected to the external grid

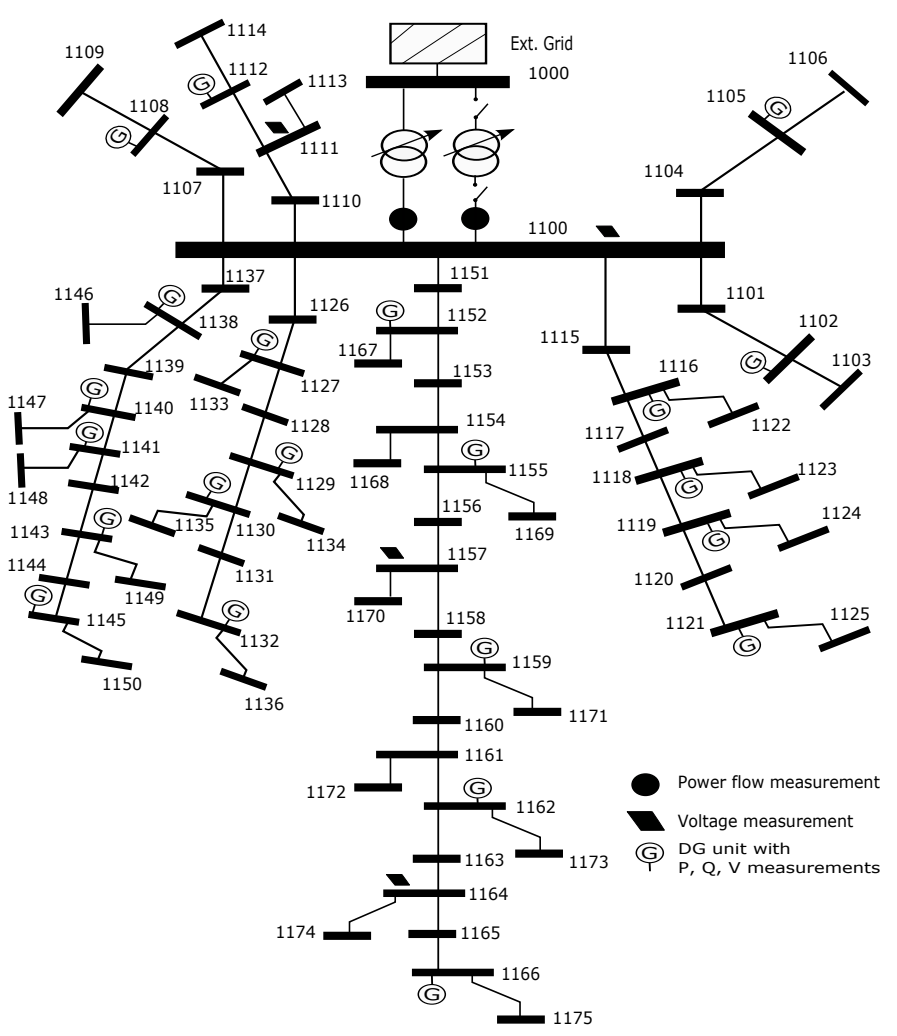

Fig. 7. 75-bus test system

through two 25-MVA 33/11 kV transformers. The external grid is represented by a Thévenin equivalent.

The network feeds 38 loads modelled as constant current (resp. impedance) for active (resp. reactive) power, and 14 with equivalent (small) induction motors.

The network also hosts 22 DGUs, of which 13 are 3MVA synchronous generators driven by $2.55-\mathrm{MW}$ turbines, and the remaining are 3.33-MVA doubly fed induction generators driven by 3-MW wind-turbines. The DGU models and parameters were taken from [23], [27], and simplified in accordance with the dynamics of interest in this work.

It is assumed that each DGUs can operate in the shaded area of the capability diagram shown in Fig. 8 [28], where $S_{\text {nom }}\left(\right.$ resp. $P_{\text {nom }}$ ) is the rated apparent (resp. active) power. The area is defined by: power factor between 0.9 and 1.0 in both under- and over-excited modes, active power smaller than nominal, apparent power $S \geq 0.1 S_{\text {nom }}$.

It is assumed that the terminal voltages and the active/reactive powers of the 22 DGUs are measured, as well as the voltage at the $11-\mathrm{kV}$ terminal of the transformer. Those measurements are received by the centralized controller every 10 seconds. The corrections $\Delta \boldsymbol{Q}_{c o r}$, obtained from (3)-(12), are sent to the DGUs with the same periodicity. The DGUs respond to these corrections within a few seconds, due to their internal dynamics.

The voltage thresholds and limits used in all simulations are given in Table I. At local level, the $V Q$ characteristics were chosen to obtain progressive reactive power changes.

In the objective function (3), $\boldsymbol{W}$ has been set to a unit matrix and the diagonal entries of $\boldsymbol{S}$ to $10^{4}$, where all voltages and 


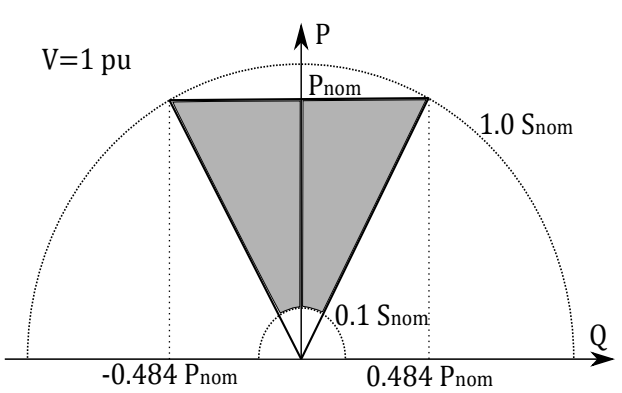

Fig. 8. DGU capability diagram; operation is allowed in the shaded area

slack variables are in per unit on the network voltage base.

TABLE I

VOLTAGE THRESHOLDS AND LIMITS

\begin{tabular}{|c|c|}
\hline $\begin{array}{c}\text { local control } \\
\text { (see Fig. 2) }\end{array}$ & $\begin{array}{c}\text { centralized control } \\
\text { (bounds in (11)) }\end{array}$ \\
\hline$V_{\min 2}^{l o c}=0.92 \mathrm{pu}$ & \\
$V_{\min 1}^{l o c}=0.97 \mathrm{pu}$ & $V_{\min }^{\text {mint }}=0.97 \mathrm{pu}$ \\
$V_{\max 1}^{\operatorname{loc}}=1.03 \mathrm{pu}$ & $V_{\max }^{c n t}=1.03 \mathrm{pu}$ \\
$V_{\max 2}^{\operatorname{loc}}=1.08 \mathrm{pu}$ & \\
\hline
\end{tabular}

The proposed controls have been implemented in RAMSES, a software for dynamic simulation in phasor mode, developed at the Univ. of Liège [29]. The local control of Fig. 3 is embedded in the differential-algebraic model of the DGU unit, while the centralized controller acts at discrete times only. The quadratic programming problem (3)-(12) is solved with the VE17AD package from Harwell [30].

\section{B. Scenario 1: Local control only}

In this first scenario, the voltages are initially within the 0.97-1.03 pu dead-band of both control levels, and all DGUs operate at unity power factor. All DGUs produce active power, which results in higher voltages as one moves away from the transformer towards the end of a feeder. The grid exports 13.7 MW and imports 6.5 Mvar, and one of the two transformers is out of service.

The assumed disturbance is a 0.05 pu drop of the Thévenin voltage at $t=30 \mathrm{~s}$. For reasons explained in Section IV-D, the transformer LTC is inoperative.

The voltage evolutions at various MV buses are shown in Fig. 9. The voltage drop is large enough to move the operating point outside the dead-band of some $V Q$ characteristics (see Fig. 2). Therefore, those DGUs with a terminal voltage lower than $V_{\min 1}^{\text {loc }}=0.97 \mathrm{pu}$ start producing reactive power right after the disturbance. The other DGUs keep operating at unity power factor. For comparison purposes, the light gray rectangle in Fig. 9 shows the range of bus voltages if there was no local control. The voltages are partly but rapidly corrected, leading to fewer buses in low voltage situation.

The reactive powers produced by various DGUs are shown in Fig. 10. It can be seen that generators 1143 and 1145 transiently produce reactive power but return to unity power factor in steady state, as they keep on operating in the deadband of their static $V Q$ characteristics. For those DGUs which

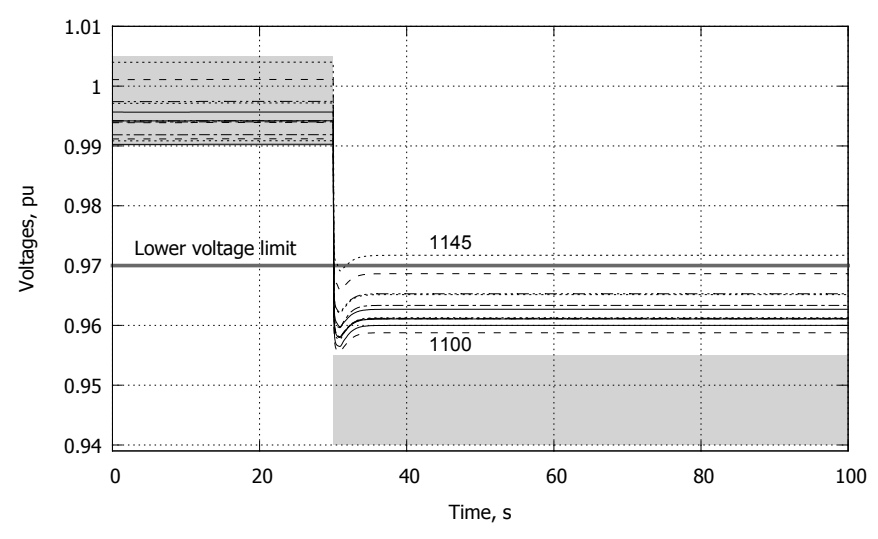

Fig. 9. Scenario 1: Voltages at a sample of MV buses

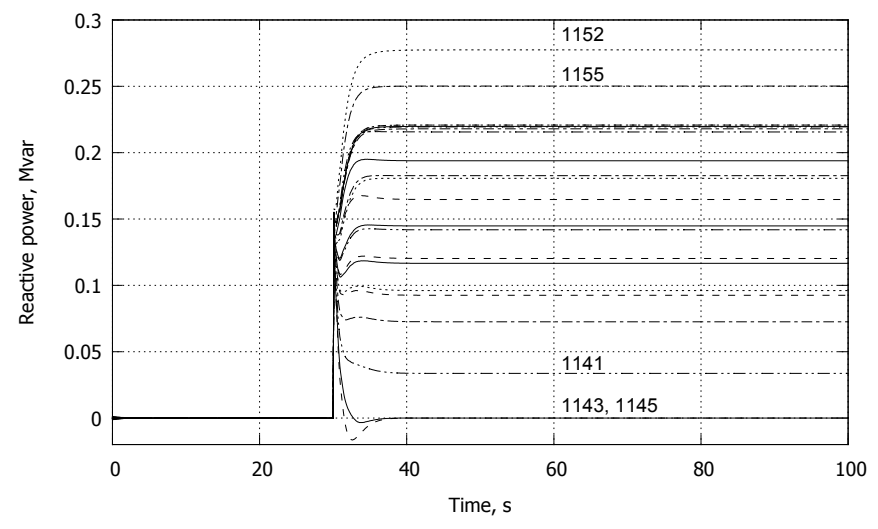

Fig. 10. Scenario 1: Reactive power produced by a sample of DGUs

eventually produce reactive power, the contribution varies with the terminal voltage and, hence, with the location.

\section{Scenario 2: Local and centralized control}

In this scenario, the operating point and the disturbance are unchanged but the centralized controller now sends the $\Delta \boldsymbol{Q}_{\text {cor }}$ corrections to the local controllers, shifting their $V Q$ characteristics until the desired reactive powers are obtained.

The bus voltages are shown in Figs. 11. Again, the corrective action of the local controllers can be assessed by comparing the light gray rectangle with the voltages reached a little before $t=40 \mathrm{~s}$, when the first $\Delta \boldsymbol{Q}_{\text {cor }}$ correction is applied. Although improved, the voltages of many buses are still below the specified lower limit.

As long as all voltages are inside the $\left[V_{\min }^{c n t} V_{\max }^{c n t}\right]$ range, the upper level does not issue any correction. It acts for the first time at $t=40 \mathrm{~s}$, after some measured voltages have been found lower than $V_{\min }^{c n t}=0.97 \mathrm{pu}$. Two control steps are enough to bring them all in the desired range, bus 1100 being just at the limit.

Figure 12 shows the variations of reactive power generations of various DGUs. From $t=30$ to $t=40 \mathrm{~s}$, the DGU reactive powers either increase or remain at zero, as in Scenario 1, while at $t=40$ and $50 \mathrm{~s}$, the centralized controller increases all of them. The contributions differ from one DGU to another, as the result of coordinated control relying on the sensitivity 


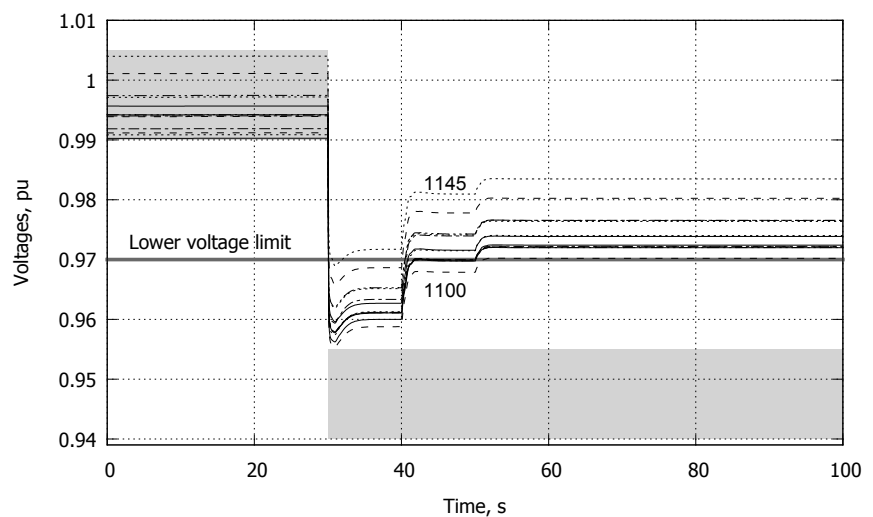

Fig. 11. Scenario 2: Voltages at a sample of MV buses

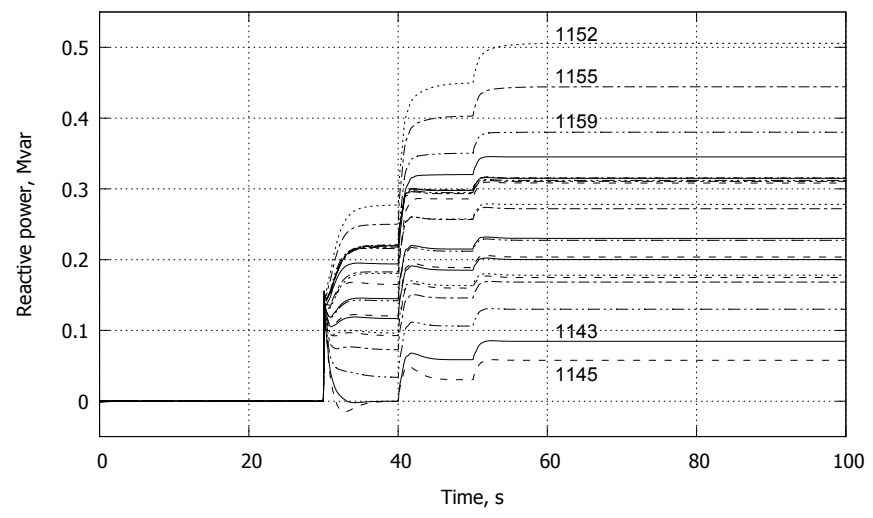

Fig. 12. Scenario 2: Reactive power produced by a sample of DGUs

matrices $\boldsymbol{S}_{V Q}$ and $\boldsymbol{S}_{Q Q}$. They would also be influenced by unequal weights in $\boldsymbol{W}$.

These corrections issued by both control levels decrease the reactive power imported by the distribution network. Given that the exported active power remains (almost) unchanged, the current in the transformer decreases, as shown in Fig. 13.

However, a branch overload problem could appear in other scenarios, for instance when the DGUs decrease their reactive power to correct an over-voltage problem. In such a case the controller would keep correcting voltages until the transformer

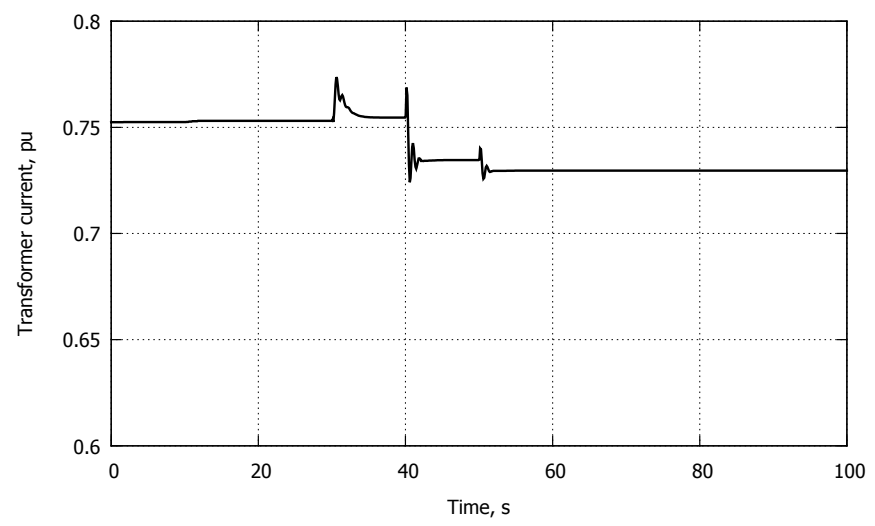

Fig. 13. Scenario 2: Current flowing in the $33 / 11 \mathrm{kV}$ transformer

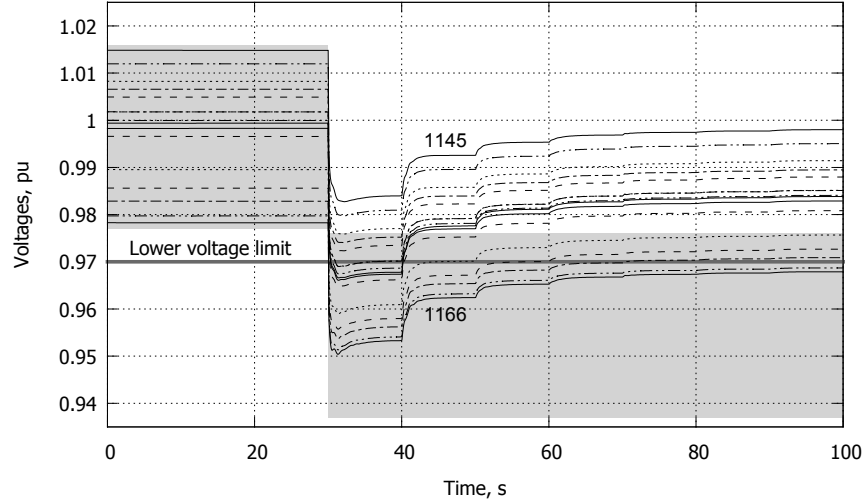

Fig. 14. Scenario 3: Voltages at a sample of MV buses

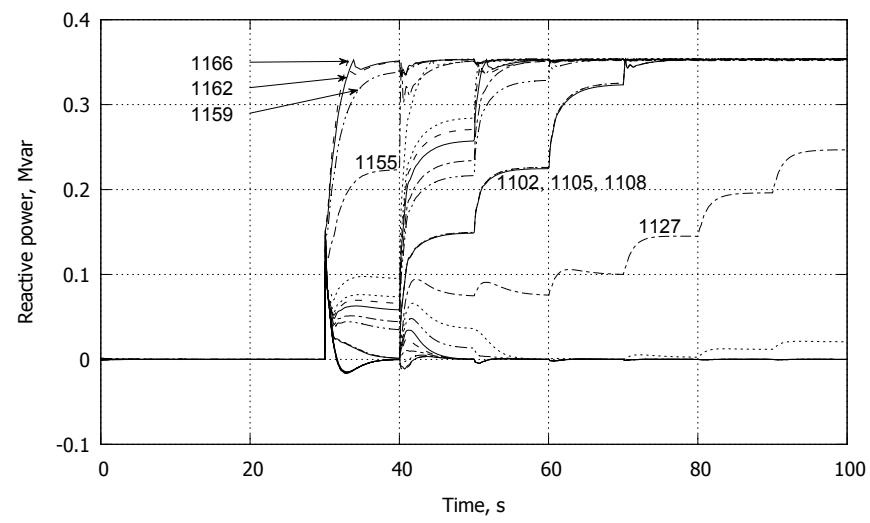

Fig. 15. Scenario 3: Reactive power produced by a sample of DGUs

current reaches its hard upper limit constraint (10). At this point, the optimisation problem (3)-(12) being infeasible, the voltage constraints (9) would be relaxed through a nonzero $\varepsilon_{2}$. This issue would be properly handled using the extended formulation of Section IV-C allowing active power corrections.

\section{Scenario 3: Local and centralized control with limited reactive reserves}

In this third scenario, a different initial operating point is considered, with a lower active power generation by the DGUs along the feeders that start at nodes 1151, 1115, 1101, 1104, 1110 and 1107. Still, a single transformer is in service. At this operating point, the MV grid receives active power from the external grid. The power flow results in lower voltages as one moves away from the transformer towards the end of the above listed feeders. As a wider range of active powers is considered, there is also a greater variety of reactive power limits.

The assumed disturbance is a 0.04 pu drop of the Thévenin voltage at $t=30 \mathrm{~s}$.

As shown in Fig. 14, some of the voltages are initially close to the lower limit and, hence, most of the buses experience under-voltage after the occurrence of the disturbance. As in the previous scenarios, the voltage fall is mitigated by the local voltage control. 


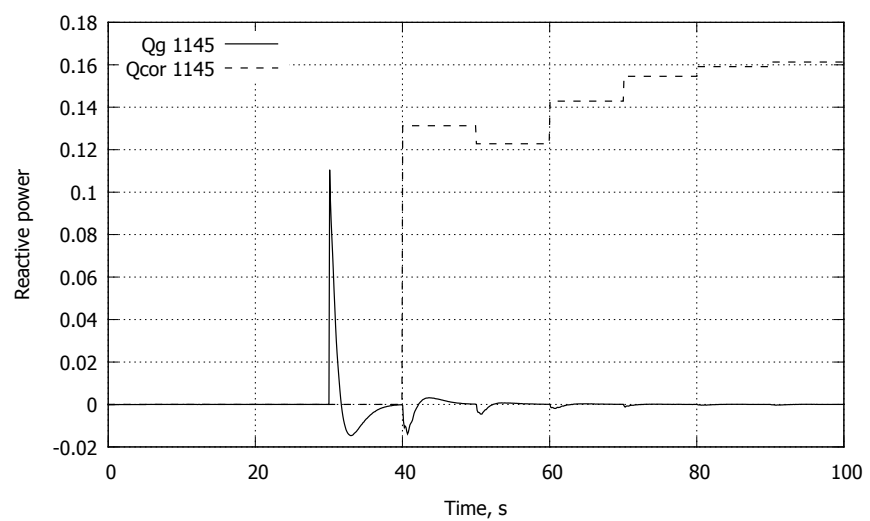

Fig. 16. Scenario 3: Reactive power corrections and DGU response

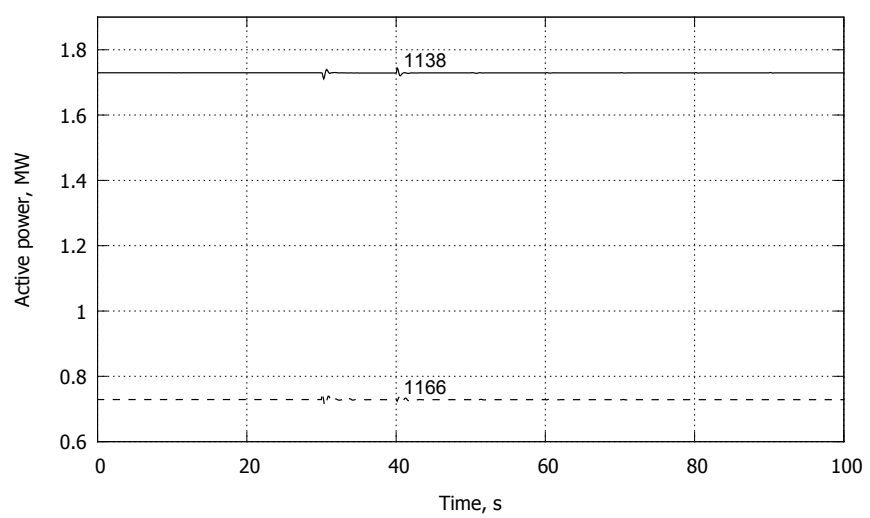

Fig. 17. Scenario 3: Active powers produced by two DGUs

The corresponding reactive power generations are shown in Fig. 15. From $t=30$ to $t=40 \mathrm{~s}$, under the effect of local control, the DGUs which undergo low voltages inject reactive power, while the others remain with a zero injection. Following the first correction, sent by the centralized controller at $t=40 \mathrm{~s}$, the grid voltages are already significantly improved. In fact, the buses with a measured voltage still below the limit at $t \simeq 49 \mathrm{~s}$ are $1166,1162,1159$ and 1157 , all located along the same, long feeder. Figure 15 shows that the DGUs at buses 1166, 1162 and 1159 have reached their maximum reactive power and, hence, cannot further help correcting the voltages. Therefore, the controller adjusts the reactive power of other DGUs, including some located in other branches, in order to raise the network voltages and correct the remaining unsatisfactory voltages. This is done smoothly in several time steps.

It is clearly seen that, in this scenario, voltages recover more slowly than in Scenario 2. Moreover, after six control steps, the voltages in the neighbourhood of bus 1166 are very close but still below the desired value. This is due to the fact that some of DGUs operate in the dead-band of their $V Q$ characteristic, while the centralized controller assumes they operate on the sloping portion, as explained in Section IV-B. Thus the DGUs of concern do not respond as expected, which leads to more control steps.

An illustration is provided in Fig. 16, comparing respec- tively the cumulated reactive power correction $Q_{c o r}$ and the effective reactive power production $Q_{g}$ of the DGU at bus 1145. Before $t=40 \mathrm{~s}$, since its terminal voltage is above $V_{\min 1}^{l o c}$, its output reactive power remains at zero, in accordance with the $V Q$ characteristic. The same holds true during the successive corrections applied by the centralized controller, which are insufficient to move the operating point on the sloping part. The situation is not known by the controller but is compensated by the closed-loop nature of MPC, which leads to a final operating point with only little violations of the voltage constraint.

In some cases, voltage violations can be corrected by acting on active power, with a higher "cost". This option was not contemplated in this work. Figure 17 shows for instance the active powers of two DGUs, which remain constant apart from tiny transients due to reactive power adjustments.

\section{E. Data communication, processing and computing times}

Each sampling period $T$ of the centralized controller can be decomposed into the following:

1) communication delay for the centralized controller corrections to be received by all DGUs;

2) dead-time ( $\simeq 2 s$ ) to wait until the DGUs and their reactive power controllers have reached steady state in response to those corrections;

3) time window $(\simeq 2 s)$ in which the measurements $\boldsymbol{V}^{m}$ and $\boldsymbol{Q}^{m}$ are locally collected and pre-filtered [10];

4) communication delay for all these measurements to be received by the centralized controller;

5) time taken by the controller to compute the corrections.

For the system of concern here, with $N=22$ DGUs and $N_{V}=4$ non-DGU bus voltage measurements, the amount of data exchanged is $N=22$ for Item 1 and $3 N+N_{V}=70$ for Item 4 (this last number includes the DGU active powers in order the centralized controller to update $Q^{\min }$ and $\boldsymbol{Q}^{\max }$ ). It is realistic to assume that the two data transfers together will not take more than one second.

As regards Item 5, on the same system, the elapsed time to solve one optimization problem (3)-(12) was found to vary between 0 and $32 \mathrm{~ms}$, depending on the number of active constraints at a given time step (obtained on a standard laptop computer with a dual-core Intel-i5 processor running at $2.27 \mathrm{GHz}$ with $4 \mathrm{~GB}$ RAM).

The sum of all the above delays amounts to $5 \mathrm{~s}$, which leaves a very ample margin with respect to the sampling period $T=10 \mathrm{~s}$.

\section{CONCLUSION}

In this paper four architectures have been outlined for voltage control of medium-voltage distribution networks by DGUs. A method pertaining to combined local and centralized controls has been presented.

The proposed control architecture consists of two levels. At the lower one, DGU reactive powers are controlled locally according to a piecewise linear static $V Q$ characteristic. This local control provides a fast response to disturbances. It 
includes a dead-band to keep DGUs operating at unity power factor as far as possible.

At the upper (centralized) level, targeting the coordination of various DGUs, MPC is at the heart of a controller computing reactive power corrections. The latter are used to adjust the $V Q$ characteristics of local controllers. To this purpose, a multi-time-step constrained optimization is solved, using a sensitivity model to predict the system behaviour. To keep this control simple and, consequently, the computational burden low, constant sensitivity matrices are used regardless of the operating point on the $V Q$ characteristics. This is made possible by the closed-loop nature and robustness of MPC.

The reported simulation results clearly show the combination of a fast but partial correction by the local controllers, followed by the smooth, coordinated control of the DGU reactive powers by the upper-level control. The latter is able to complement the actions taken locally, taking into account the various DGU reactive power reserves as well as the cost and impact of DGU reactive power adjustments.

An immediate extension of this work consists of investigating the performance of the combined control scheme steering only a subset of DGUs, the rest of them being under local control only. This amounts to mixing the architectures shown in Figs. 1.a and 1.c, respectively.

A further extension would be to deal with three-phase models to cope with grids exhibiting power/voltage imbalances.

\section{ACKNOWLEDGMENT}

Work supported by Public Service of Wallonia, Department of Energy and Sustainable Building, within the framework of the GREDOR project.

The valuable discussions with Dr. Mevludin Glavic are gratefully acknowledged.

\section{REFERENCES}

[1] Q. Gemine, E. Karangelos, D. Ernst, and B. Cornelusse, "Active Network Management: Planning under Uncertainty for Exploiting Load Modulation," in 2013 IREP Symposium Bulk Power System Dynamics and Control - IX Optimization, Security and Control of the Emerging Power Grid, IEEE, 2013.

[2] V. A. Evangelopoulos, P. S. Georgilakis, and N. D. Hatziargyriou, "Optimal Operation of Smart Distribution Networks: A Review of Models, Methods and Future Research," Electric Power Systems Research, 2016.

[3] P. Kotsampopoulos, N. Hatziargyriou, B. Bletterie, and G. Lauss, "Review, Analysis and Recommendations on Recent Guidelines for the Provision of Ancillary Services by Distributed Generation," 2013 IEEE International Workshop on Inteligent Energy Systems (IWIES), 2013.

[4] "Integrating Distributed Generation Using Decentralised Voltage Regulation," IEEE Power and Energy Society General Meeting, 2010.

[5] T. Sansawatt, L. F. Ochoa, and G. P. Harrison, "Smart Decentralized Control of DG for Voltage and Thermal Constraint Management," IEEE Trans. on Power Systems, vol. 27, no. 3, 2012.

[6] B. A. Robbins, C. N. Hadjicostis, and A. D. Dominguez-Garcia, "A TwoStage Distributed Architecture for Voltage Control in Power Distribution Systems," IEEE Trans. on Power Systems, vol. 28, no. 2, 2013.

[7] P. N. Vovos, A. E. Kiprakis, A. R. Wallace, and G. P. Harrison, "Centralized and Distributed Voltage Control: Impact on Distributed Generation Penetration," IEEE Trans. on Power Systems, vol. 22, no. 1, 2007.

[8] A. Vaccaro and A. F. Zobaa, "Voltage regulation in active networks by distributed and cooperative meta-heuristic optimizers," Electric Power Systems Research, vol. 99, 2013

[9] A. Seack, J. Kays, and C. Rehtanz, "Time Series Based Distribution Grid Planning Approach with Decentralised Voltage Regulation," in IEEE Power Systems Computation Conference, 2014.
[10] G. Valverde and T. Van Cutsem, "Model Predictive Control of Voltages in Active Distribution Networks," IEEE Trans. on Smart Grid, vol. 4, no. 4, 2013.

[11] H. Soleimani Bidgoli, M. Glavic, and T. Van Cutsem, "Model Predictive Control of Congestion and Voltages Problems in Active Distribution Networks," Proc. of CIRED Workshop, no. June, 2014.

[12] H. Soleimani Bidgoli, M. Glavic, and T. Van Cutsem, "RecedingHorizon Control of Distributed Generation to Correct Voltage or Thermal Violations and Track Desired Schedules," 19th Power System Computation Conference (PSCC'16), Genoa, Italy, June 2016.

[13] J. M. Maciejowski, Predictive Control With Constraints. Prentice-Hall, 2002.

[14] S. J. Qin and T. A. Badgwell, "A survey of industrial model predictive control technology," Control Engineering Practice, vol. 11, no. 7, 2003.

[15] S. W. Alnaser and L. F. Ochoa, "Advanced network management systems: A risk-based AC OPF approach," IEEE Transactions on Power Systems, vol. 30, 2015.

[16] M. Farina, A. Guagliardi, F. Mariani, C. Sandroni, and R. Scattolini, "Model Predictive Control of Voltage Profiles in MV Networks with Distributed Generation," Control Engineering Practice, vol. 34, 2015.

[17] Y. Chistyakov, E. Kholodova, K. Netreba, A. Szabo, and M. Metzger, "Combined Central and Local Control of Reactive Power in Electrical Grids with Distributed Generation," 2012 IEEE International Energy Conference and Exhibition, ENERGYCON 2012, 2012.

[18] N. Jayasekara, P. Wolfs, and M. A.S. Masoum, "An optimal management strategy for distributed storages in distribution networks with high penetrations of pv," Electric Power Systems Research, vol. 116, 2014.

[19] J. Backes, C. Schorn, and H. Basse, "Cost-Efficient Integration of Dispersed Generation Using Voltage Dependent Reactive Power Control," in Proc. of CIRED Workshop, no. June, 2010.

[20] K. Turitsyn, P. Sulc, S. Backhaus, and M. Chertkov, "Options for Control of Reactive Power by Distributed Photovoltaic Generators," Proceedings of the IEEE, vol. 99, no. 6, 2011.

[21] T. Kerber, G. Kerber, M. Werner, M. Finkel, and M. Wiest, "Decentralized Voltage Control in $20 \mathrm{kV}$ Distribution Grids by Controlling Reactive Power of Inverters," in Proc. of CIRED Workshop, 2014.

[22] M. Rylander, H. Li, J. Smith, and W. Sunderman, "Default Volt-Var Inverter Settings to Improve Distribution System Performance," In Proc. IEEE Power Engineering Society General Meeting, 2016.

[23] K. Clark, N. W. Miller, and J. J. Sanchez-Gasca, "Modeling of GE wind turbine-generators for grid studies," General Electric International, Version 4.5, USA, 2010

[24] G. Valverde and J. Orozco, "Reactive power limits in distributed generators from generic capability curves," in Proc. IEEE PES 2014 General Meeting, (Washington DC.), 2014.

[25] D. Q. Mayne, J. B. Rawlings, C. V. Rao, and P. O. Scokaert, "Constrained model predictive control: Stability and optimality," Automatica, vol. 36 , no. 6 , pp. 789-814, 2000 .

[26] "United Kingdom Generic Distribution Network (UKGDS)," Available: http://sedg.ac.uk.

[27] J. D. Hurley, L. N. Bize, and C. R. Mummert, "The Adverse Effects of Excitation System Var and Power Factor Controllers," IEEE Trans. on Energy Conversion, vol. 14, no. 4, 1999.

[28] CENELEC, "CLC/TS 50549-2 Requirements for Generating Plants to be Connected in Parallel With Distribution Networks - Part 2: Connection to a MV Distribution Network," technical specification, 2015.

[29] P. Aristidou, S. Lebeau, and T. Van Cutsem, "Power System Dynamic Simulations Using a Parallel Two-Level Schur-Complement Decomposition," IEEE Trans. on Power Systems, 2016.

[30] "HSL. A Collection of Fortran Codes for Large Scale Scientific Computation . [Online]," Available: http://www.hsl.rl.ac.uk.

Hamid Soleimani Bidgoli ( $S^{\prime}$ 13) received his B.Sc. degree in Electrical Engineering from IUST, Tehran, Iran and his M.Sc. in the same field from University of Tehran, Iran, in 2008 and 2010, respectively. It was followed by three years of work in the power generation sectors. He is currently pursuing his $\mathrm{PhD}$ degree at University of Liège, Belgium. His main scientific interests are active network management of distribution networks, real-time control, and demand side management.

Thierry Van Cutsem (F' 05) received the M.Sc. and Ph.D. degrees from the University of Liège, Belgium, in 1979 and 1984, respectively. He is currently a Research Director of the Fund for Scientific Research (FNRS) and an Adjunct Professor at the Dept. of Electrical Engineering and Computer Science at the same university. His research interests include power system dynamics, security, monitoring, control, and simulation. He is currently the Past Chair of the IEEE PES Power System Dynamic Performance Committee. 